\title{
ESTIMATION TECHNIQUES USED IN STUDIES OF COPEPOD POPULATION DYNAMICS - A REVIEW OF UNDERLYING ASSUMPTIONS
}

\author{
Dag L. Aksnes, Charles B. Miller, Mark D. Ohman \& Simon N. Wood
}

SARSIA

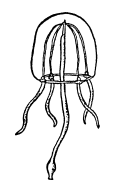

Aksnes, Dag L., Charles B. Miller, Mark D. Ohman \& Simon N. Wood 199712 22. Estimation techniques used in studies of copepod population dynamics - a review of underlying assumptions. Sarsia 82:279-296. Bergen. ISSN 0036-4827.

The literature on zooplankton population dynamics provides more estimation techniques than reliable estimates of population parameters. In this review we show how different techniques relate to each other in terms of underlying models and assumptions. There are two main routes to parameter estimates. The vertical approaches utilize the stage-structure of samples taken at the same point in time. They require assumptions about the constancy in the parameters, but relax assumptions concerning advective influence. The horizontal approaches utilize information provided by the stage structure within samples, as well as information on temporal changes in abundance. They relax assumptions about the constancy of parameters, but advective influences may be introduced. Of the horizontal methods, variants of what are commonly termed cohort methods have been widely used. These provide mathematical simplicity, but are based on more restrictive assumptions than methods fitting predescribed models (delay differential, partial differential equation, and matrix models of population dynamics). Because the quality of estimates, such as of mortality rates, depends upon the quality of the data, some sampling considerations in regard to advection and biases in estimating stage composition are discussed. It is important to reduce the number of parameters to be estimated from census data; thus we review some techniques for independent measurement of stage durations and birth rates. Finally, we provide some general recommendations for future studies.

Dag L. Aksnes (present address), University of Bergen, Department of Fisheries and Marine Biology, Bergen High-Technology Center, N-5020 Bergen, Norway-Dag L. Aksnes (research address) \& Mark D. Ohman, Marine Life Research Group 0227, Scripps Institution of Oceanography, U.C. San Diego, La Jolla, CA 92093-0227, USA - Charles B. Miller, College of Oceanographic and Atmospheric Sciences, Oregon State University, Corvallis, OR 97331-5503, USA - Simon N. Wood, NERC Centre for Population Biology, Imperial College at Silwood Park, Ascot, Berkshire SL5 7PY, United Kingdom.

KEYwORDs: Review; population dynamics; copepods; estimation techniques.

'The mismatch between populations and the models is not a fault of the animals.' - Edmondson 1993

\section{INTRODUCTION}

Research on population dynamics of marine zooplankton is carried out because of their important roles as grazers, prey and competitors in a globally critical habitat, the marine pelagial. Zooplankton often have important influence on other pelagic trophic levels. The longer time scales of the processes involved in zooplankton growth, relative to those involved in growth of phytoplankton, often require that characteristics like recruitment and mortality have to be addressed in order to estimate zooplankton production. Also, in ecosystem studies concerned with elemental fluxes the central importance of zooplankton mortality patterns has become clear (e.g. FASHAM 1993).
A population dynamical approach is useful in calculation of secondary production and for testing of hypotheses concerning effects of such factors as temperature and food availability on zooplankton growth. It is also useful in descriptive life-history studies. In the simplest form, population dynamics are described by time series of population size. However, description of the underlying vital rates is the only way to achieve mechanistic understanding of population processes.

The average properties of a population may not be good predictors of the properties of the survivors in that population. Individual-based approaches that incorporate variability in parameters such as fecundity, development times, growth rates and risk of mortality may be necessary to successfully model and under- 
stand population dynamics (DeANGelis \& Gross 1992; Tyler \& Rose 1994; AKsnes \& OHMAn 1996). However, because of the challenges associated with obtaining even the mean or median parameter values in zooplankton population research, we focus on methods for obtaining such estimates of central tendencies. Furthermore, we restrict ourselves to review of the copepod literature. Many estimation techniques have been suggested. Rather than assessing their performance by simulations, we will focus on the models and assumptions underlying the techniques. A main aim is to show the connections between different techniques. Hence, we use a common symbol notation throughout the paper (Table 1).

There are many crucial steps in population dynamics research that influence the quality of parameter estimates. The first requirement is collection of unbiased census data, and unfortunately the marine environment presents several challenges for sampling planktonic populations. Most zooplankton species have wide geographical distributions, and normally sampling has to be limited to restricted parts of those ranges. The extent to which sampled individuals represent a homogeneous population, several subpopulations changing at different dynamical rates, or populations exchanging through the boundaries of the sampled domain is fundamental to the interpretation of the observed dynamics. Investigators should generally seek to minimize the influence of migration and advection, as required by most techniques currently available for estimating demographic parameters (see MANLY 1990; WoOd \& Nisbet 1991; Wood 1994). Sophisticated estimation methods cannot compensate for poor data (but similarly, poor methods will waste good data), so before discussing different estimation techniques, we consider two important aspects of zooplankton sampling.

Individual zooplankters generally cannot be aged, so that age must be described indirectly by development stages or age-size relationships. Unfortunately, this introduces an additional set of parameters, such as stage durations, that must be estimated. Inclusion of independent (i.e., not extracted from the census data), but accurate, measurements of stage durations can increase the reliability of recruitment and mortality estimates. Likewise, in studies encompassing the earliest life stages, measurements of egg production (i.e., birth rate) can be used to improve the quality of mortality estimates. Hence, we will emphasize the importance of combining census-based and experiment-based estimation techniques in approaching the dynamics of populations, and we review some techniques for experimental measurement of stage durations and birth rate.

\section{SAMPLING CONSIDERATIONS}

We address two sampling concerns that require particular attention in population dynamics research on copepods. The first is the potential impact of advection on observed dynamics. Ignorance of this source of forcing may lead to time series containing misleading information about death and birth processes. The second is unequal sampling efficiency for the different development stages. Over- and underestimation of the abundances of one development stage relative to another are sources of bias in population dynamical analyses. Such biases can arise due to advection and behavior as well as to sampling gear performance.

\section{ADVECTION}

By definition, the 'plankton' are influenced by fluid motions. The quantitative impact of advection on zooplankton dynamics was addressed by КетснUм (1954) and recently by AKsnes \& Blindheim (1996). Some marine environments with restricted circulation, such as certain fjords and bays, may reduce the influence of advection on zooplankton dynamics (LANDRY 1978; Aksnes \& Magnesen 1983; Ohman 1986). Even fjords, however, experience flushing events (OsGOoD \& FROST 1996) and such localities are not a warranty against advective impact. As demonstrated below, the advective influence on zooplankton dynamics depends on the spatial scale of the sampling area, the biological rates of change, and the velocity of the water movements.

The dynamics of a zooplankton stock that is distributed homogeneously over a habitat volume $V$, corresponding to the geographical target of our investigation, may be expressed:

$$
\mathrm{d} n / \mathrm{d} t=(b-d) n-\varepsilon n+t n_{b}
$$

where $n$ is the abundance of the local stock within the habitat volume $V, n_{b}$ is the abundance of the stock surrounding the investigated volume, and $b, d, \varepsilon$ and $t$ are the birth, mortality, emigration and immigration rates. If 'migrations' are dominated by horizontal water transport rather than by swimming, we may assume (AKSNES \& al. 1989):

$$
\varepsilon=\imath=u C / V
$$

where $u$ is the advective rate across the boundary areas $(C)$ of the habitat volume $(V)$. Insertion of Eq. 2 into Eq. 1 gives:

$$
\mathrm{d} n / \mathrm{d} t=(b-d) n+(u C / V)\left(n_{b}-n\right)
$$


Table 1. Explanation of symbols. d.l. means dimensionless. Although the units of birth rates are normally given as $\mathrm{d}^{-1}$, this unit should primarily be used when the birth is given per capita (i.e. relative to all members of the population such as in $r$ ). In zooplankton studies birth rate is often defined as eggs released per adult female per day (denoted egg $\mathrm{f}^{-1} \mathrm{~d}^{-1}$ below) which is a different measure. Note that the egg-ratio method of EDMONDSON (1960) was originally derived for parthenogenic egg-carrying populations where all individuals were females. The egg-ratio was expressed all eggs:all females (juveniles and adults) and the Edmondson birth rate can therefore be considered to be a per capita rate.

\begin{tabular}{|c|c|c|}
\hline Symbol & Explanation & Unit \\
\hline$A_{i}$ & Area under the frequency curve of stage $i$ & ind $d$ \\
\hline$a_{i}$ & Stage duration of stage $i$ & d \\
\hline$\alpha_{i}$ & Age of an individual at the time when stage $i$ is entered & d \\
\hline$B_{i}$ & Temporal mean of the frequency curve of stage $i$ & d \\
\hline$b$ & Instantaneous birth rate (defined relative to all individuals) & $d^{-1}$ \\
\hline$b_{f}$ & Birth rate (defined relative to adult females) & $\operatorname{egg~} f^{-1} d^{-1}$ \\
\hline$\beta$ & Finite birth rate $\beta=b(\exp (r)-1) / r$ & d.1. \\
\hline$\beta_{\mathrm{E}}$ & Edmondson finite birth rate $\left(\beta_{\mathrm{E}}=\mathrm{E}_{\mathrm{r}} / \mathrm{a}_{1}\right)$ & $d^{-1}$ \\
\hline$C$ & The area of the boundaries of a zooplankton habitat & $\mathrm{m}^{2}$ \\
\hline$d$ & Instantaneous death rate (defined relative to all individuals) & $d^{-1}$ \\
\hline$d_{i}$ & Instantaneous death rate of stage i & $d^{-1}$ \\
\hline$d(x, t)$ & Instantaneous death rate of individuals of age $\mathrm{x}$ at time $\mathrm{t}$ & $d^{-1}$ \\
\hline $\mathrm{E}_{\mathrm{r}}$ & Edmondson egg ratio (all eggs and individuals are females) & egg ind ${ }^{-1}$ \\
\hline$\varepsilon$ & Emigration rate & $\mathrm{d}^{-1}$ \\
\hline$f_{i}$ & The ratio of the abundances of two sucessive stages $\left(f_{i}=n_{i} / n_{i+1}\right)$ & d.1. \\
\hline$G_{i}$ & Probability of surviving and growing from stage $i$ to $i+1$ over the time period $t_{j}$ to $t_{j+1}$ & d.1. \\
\hline$g_{i}(t)$ & A function describing the shape of recruitment to stage i over time $\left(R_{i}(t)=T_{i} g_{i}(t)\right)$ & d.1. \\
\hline$H_{i}$ & The temporal median (see text) & d \\
\hline$i$ & Stage index ( $i=1$ is egg stage and $i=q$ is adult stage) & - \\
\hline$j$ & Sampling occasion number $\mathrm{j}$ & - \\
\hline$i+1 . . q$ & Index for the combined stage including $i+1, i+2, \ldots ., q$ & - \\
\hline$\imath$ & Imigration rate & $d^{-1}$ \\
\hline$M_{i}(t)$ & Maturation rate from stage $i$ at time $t, M_{i}(t)=R_{i+} 1(t)$ & ind $\mathrm{d}^{-1}$ \\
\hline$m_{i}(t)$ & Molting rate for stage $i$ (i.e. molting from $i$ to $i+1$ ) at time $t$ & $d^{-1}$ \\
\hline$\mu_{i}$ & Time for peak recruitment to stage $i$ & d \\
\hline$n$ & Total population size (ind $\mathrm{m}^{-3}$ in the case of Eq. 1 and 3) & ind \\
\hline$n b$ & Boundary abundance & ind $\mathrm{m}^{-3}$ \\
\hline$n_{i}$ & Number in stage $i$ & ind \\
\hline$n_{f}$ & Number of adult females (i.e. females in stage $q$ ) & f \\
\hline$n(x, t)$ & Number of age $x$ at time $t$ & ind \\
\hline$P_{i}$ & Probability of surviving and remaining in stage $i$ over the time period $t_{j}$ to $t_{j+1}$ & d.1. \\
\hline$p_{i}$ & Relative abundance of a stage (stage proportion) & d.1. \\
\hline$q$ & Index for the adult stage & - \\
\hline$R_{i}(t)$ & Recruitment rate to stage $\mathrm{i}$ at time $\mathrm{t}, R_{i}(t)=M_{i-1}(t)$ & ind $\mathrm{d}^{-1}$ \\
\hline$r$ & Instantaneous rate of increase, $r=b-d$ & $d^{-1}$ \\
\hline$\rho_{i}$ & The ratio of the abundances of two consecutive stages $\mathrm{n}_{i} / \mathrm{n}_{i+1}$ & d.1. \\
\hline$S_{i}$ & Stage specific survival $S_{i}=\exp \left(-d_{i} a_{i}\right)$ & d.1. \\
\hline$T_{i}$ & Total recruitment to stage $i$ (of a cohort) & ind \\
\hline$t$ & Time & d \\
\hline$t j$ & Time at sampling occasion $\mathrm{j}$ & d \\
\hline$V$ & The habitat volume considered in an investigation & $\mathrm{m}^{3}$ \\
\hline$u$ & The advective rate across the habitat boundary A & $\mathrm{m} \mathrm{d}^{-1}$ \\
\hline$x$ & Age & d \\
\hline
\end{tabular}


Although this simplified expression of the population dynamics ignores age distribution and spatial distribution of the animals within the habitat, and lumps the hydrodynamics into a single coefficient, it is appropriate for a discussion of the potential impact of advection on zooplankton dynamics.

Eq. 3 expresses the dynamics of the population $(\mathrm{d} n /$ $\mathrm{d} t)$ as a function of the birth and death rates $(b$ and $d)$, and three parameters governed by hydrodynamics and the spatial scale of our investigation $(u, C$ and $V)$. If the physical-spatial coefficient $u C / V$ is much greater than the biological coefficients $b$ and $d$, we have a stock whose dynamics is dominated by advection rather than biology and vice versa. Note that in the case where $b$ and $d$ are equal, even minor advective influence may be decisive for the local change in number of individuals, although local appearance and removal of individuals are dominated by births and deaths. Then temporal changes in $n$ will not carry any information about the death and birth rates at all. Furthermore, if $n_{b}$ is equal to $n$ one might think that the local population is uninfluenced by advection. If $u C / V$ is large, however, appearance and removal of individuals will be dominated by the advective flux rather than by births and deaths. In order to judge the influence of advection it is appropriate to relate the advective rate to both the death rate and the birth rate. Hence if

$$
b>>u C / V \text {, and } d>>u C / V
$$

are satisfied, we may obtain time series dominated by biology rather than advection Three different ways of reducing the advective influence are indicated by Eq. 4 : 1 . Reduce the boundary surface $(C)$ against other systems. For a fjord-like system, this simply means that the fjord with the smallest possible cross-section area above the sill is the best site (unless this decrease in border area is compensated by a corresponding increase in the water currents across the border area). 2. Reduce the exchange rate $(u)$ with other systems. 3 . Increase the volume $(V)$ to be covered by sampling so that: $V>>u C / d$ and $V>>u C / b$. Not explicitly expressed in Eq. 4, a fourth way to reduce the advective impact is to move the sampling domain $(V)$ at the same rate as the advective rate $(u)$, i.e. to use a Lagrangian sampling design to follow the water mass containing the population. This was attempted by Cushing \& Tungate (1963) in their study of the development of a Calanus finmarchicus patch in the North Sea and HERON (1972) in his study of the salp Thalia democratica.

Behavioral adaptations promoting retention within geographical areas seem important in marine zooplankton (Sinclair 1988; KAARTVEDT 1993), and such behavior may decrease the actual influence of advection in population dynamics research. Vertical migration in regions of vertical current shear is probably the most important aid a zooplankter has to facilitate retention. Hence, assessment of vertical migration patterns should be integrated with investigation of zooplankton population dynamics. Such knowledge can be utilised in order to correct more accurately for advective influence. As will be pointed out later on, stage specific migration patterns also represent a source of bias in the sampling of the population stage structure and assessment of stage durations.

Despite retention mechanisms, it will often be costprohibitive to account appropriately for advective influence. In such cases the vertical approach to mortality estimation may be useful (Mullin \& BRooks 1970; Aksnes \& Ohman 1996). This technique utilises the observed stage composition within samples rather than changes in abundances over time, and is therefore more robust against advective influence than the horizontal techniques assuming closed populations. As emphasized later, however, this gain in robustness has a cost in terms of more restrictive assumptions concerning the underlying population processes.

\section{BIASED STAGE COMPOSITION}

All estimation techniques require data in which the sampling bias is equal across the full range of stages. MILLER \& TANDE (1993) pointed out that the abundances of the early development stages are often likely to be underestimated relative to those of later copepodite stages. For nets towed from deep to shallow, the interaction between deepening vertical distributions of successive stages and clogging may give rise to such biased stagestructure. The younger, shallower-living stages are undersampled relative to older ones because the filtration efficiency drops before the rising net reaches them. Hence, filtration efficiency should be monitored continuously during sampling. Pumps can be an effective alternative (MILLER \& JUDKINS 1981), but are often not suitable for highly motile stages (Mullin \& BRooKs 1976) or for soft-bodied, gelatinous organisms.

In addition to gear performance, the interaction between water transport and vertical distribution may also cause bias in the sampled stage composition. Generally, water velocities are higher in shallow than in deeper water. Hence, younger stages confined to the surface layer are likely to experience greater dispersion than the older stages occupying deeper strata. Depending on time and the geographical sampling location (relative to spawning patterns), these conditions may give rise to either under- or overestimation of the younger stages. Topography is also important in shaping local zooplankton dynamics. Deep basins in otherwise shal- 
low areas are likely to facilitate aggregation of the older stages (Herman \& al. 1991) which may cause skewed stage composition in depth-integrated samples. For a fjord population of Calanus finmarchicus, AKsNes \& MAGNESEn (1983) found that time series obtained at the deepest sampling location gave rise to biased abundance estimates, while stratified sampling that accounted for the depth-dependent distribution of the development stages increased the accuracy of the estimates. In the limnological literature aggregation of individuals over deeper water is a widespread phenomenon, frequently described as shore avoidance (GLIwICZ \& RYKowsKa 1992). The reasons for this phenomenon can be several; active avoidance of shallow areas as the individuals age, passive aggregation caused by the interaction of water movement and vertical migration, or simply higher predation pressure in the shallower areas. Again, assessment of vertical distribution and migration patterns seems necessary to resolve such questions.

\section{THE POPULATION PARAMETERS AND TECHNIQUES FOR DIRECT MEASUREMENT}

Changes in numbers over time in a closed population occur from the difference between the rate of recruitment and rate of mortality. For organisms that develop through a sequence of discrete developmental stages (e.g., planktonic crustaceans), the recruitment rate and death rate typically vary with stage. For a stage-structured population, changes in the abundance $\left(n_{i}\right)$ of a stage $i$ may be described as the recruitment rate $\left(R_{i}(t)\right)$ into the stage minus the rate of maturation $\left(M_{i}(t)\right)$ to the next stage $i+1$ minus the mortality within the stage (where $d_{i}(t)$ is the instantaneous mortality rate):

$$
\frac{\mathrm{d} n_{i}}{\mathrm{~d} t}=R_{i}(t)-M_{i}(t)-d_{i}(t) n_{i}(t)
$$

where the number recruiting to stage $i+l$ is equal to the number leaving stage $i$ :

$$
\begin{gathered}
R_{i+1}(t)=M_{i}(t), \\
M_{i}(t)=R_{i}\left(t-a_{i}(t)\right) \exp \left[-\int_{t-a_{i}}^{t} d_{i}(x) \mathrm{d} x\right]\left(1-\frac{\mathrm{d} a_{i}(t)}{\mathrm{d} t}\right)
\end{gathered}
$$

Hence, the number leaving a stage depends on the stage duration $\left(a_{i}(t)\right)$ and the death rate $\left(d_{i}(t)\right)$ as well as the number entering. The recruitment to the first $(i=1$, commonly the egg) stage and the number leaving the final stage $(i=q)$, however, can be expressed:

$$
\begin{gathered}
R_{1}(t)=b_{f}(t) n_{f}(t) \\
R_{q+1}(t)=0
\end{gathered}
$$

where $b_{f}$ is the birth rate of the average adult female and $n_{f}$ is the number of adult females. Because birth rates, recruitment rates, and mortality rates typically vary over time, measurements and estimation procedures should ideally accommodate both time- and stage-specific rates. The above delay differential equation model (Eqs 5a-d) of stage abundance accounts for such variability (note, however, that it does not account for individual variability in stage duration or mortality risk). As discussed later, one way of estimating population parameters is to fit this kind of model to stage frequency data. First, however, we will consider the possibility of direct measurement of some of the parameters as all estimation procedures greatly benefit from a reduction in the number of parameters to be estimated indirectly.

Considerable effort in recent decades has gone into analysis of the factors controlling variability in birth (i.e. the $b_{f}$ or other related birth rate parameters, see Table 1), development and growth rates in zooplankton populations. An assumption underlying such efforts is that zooplankton population dynamics are influenced primarily by variability in birth and development, rather than mortality. However, some evidence (IANORA \& ButTino 1990; OHMAN \& WoOd $1995)$ challenges this assumption and suggests that mortality rates of natural populations may show greater variability. Furthermore, adaptive antipredator behavior generates variability in vital rates such as egg production and development time (AKSNES 1996). It has become clear that the mortality rate terms in population dynamics studies has seen far too little attention in the past. This is partly due to the facts that mortality rates usually cannot be measured directly and that no standard estimation technique has emerged. Birth rate and stage durations, however, are quantities that can be measured more directly.

\section{BIRTH RATE}

As the initial input term to actively growing populations, the birth rate term must be measured carefully and interpreted correctly. Mortality estimation will greatly benefit from accurate birth rate measurements, because it is important to restrict the number of parameters to be estimated from census data. In this context it is important that the birth rate measure and the estimation model are compatible. In a population dynamics model combining adult females and males into a common 
stage $\mathrm{q}$, the birth rate per adult $\left(b_{q}\right)$ may be needed rather than $b_{f}$ (which is appropriate when adult females and males are separated). Similarly, in models where all individuals make up one stage, the per capita birth rate is expressed (the $b$ of the instantaneous growth rate $r$ ). Hence, if birth rate measurements are carried out to support mortality estimation, it is important that accurate abundance measurements of the appropriate category of animals (adult females, adults, total numbers, etc.) are carried out in addition to the actual number of births (i.e. egg production).

\section{Egg-carrying life histories}

For species that carry eggs as an attached egg mass, Edmondson's egg ratio method (EDMONDSON 1960, 1993) can be used to approximate the birth rate $(b)$. This approach applies only when the population is reproducing continuously (at a constant rate) and approximates a stable age distribution. A finite rate of daily egg hatching per female $\left(\beta_{E}\right)$ was expressed by EdMONDSON (1960) as:

$$
\beta_{E}=\frac{E_{r}}{a_{1}}
$$

where $E_{r}$ is the egg ratio (all eggs divided by all females, both juveniles and adults) and $a_{1}$ is the embryonic duration (egg stage duration). Embryonic duration is usually a well-defined function of temperature (e.g. MCLAREN $\&$ al. 1989). However, Eq. 6 assumes a uniform age distribution of eggs, which occurs only when there is no egg mortality, and results in a biased estimate of egg hatching rate when mortality occurs. THRELKELD (1979) illustrated that where the egg age distribution can be assessed by morphological assessment of stage of embryonic development, an improved measure of egg hatching rate can be obtained.

Several formulae have been proposed to relate the Edmondson egg-ratio $E_{r}$ (and the finite birth rate $\beta_{E}$ ) to the instantaneous birth rate, $b$, (see Gabriel $\&$ al. 1987; BRETT \& al. 1992; EdMONDSON 1993). PALOHEIMo (1974) showed that:

$$
b=\frac{\ln \left(1+E_{r}\right)}{a_{1}}
$$

It should be noted that Eqs 6 and 7 were primarily formulated for populations consisting of egg-carrying females and, as discussed above, that application to other populations requires some care regarding the definition of birth rate. Eq. 7 appears most appropriate when the sampling interval is close to the embryonic duration (GABRIEL \& al. 1987). At longer sampling intervals, TAYLOR \& SLATKIN's (1981, their eq. 7) method may be more appropriate in the case where juveniles and adults can be analysed separately, and where juvenile development time can be measured accurately.

Incubations of adult females can also be used to measure directly the daily rate of egg production of egg brooding species, although when this rate is low, representative temperature and food conditions must be maintained for rather lengthy incubations.

\section{Broadcast spawning life histories}

As Edmondson (1993, p. 76) stated, 'the fact that some species of copepods, especially marine ones, do not carry eggs eliminates them from an egg ratio analysis.' Although the egg ratio method has been applied to broadcast-spawning copepods that release eggs freely into the water column, it is not appropriate for such a life history because the mortality experienced by eggs while suspended in the water column can strongly bias the estimated rate of egg hatching (PETERSON \& KiMmereR 1994). From the high egg mortality rates frequently observed (LANDRY 1978; Uye 1982; KiøRBOE \& AL. 1988; Peterson \& Kimmerer 1994; Kiørboe \& Nielsen 1994), we suggest that observed egg abundances of eggs suspended in the water column arising from broadcast spawners should be used in the estimation of mortality rather than of fecundity, and that egg production rates should be measured in appropriate incubations. Eq. 5c can then be used to estimate the recruitment to the egg stage. This quantity (together with embryonic duration) can then enter one of the methods described later to facilitate mortality estimation.

\section{Influence of mortality on birth rate measurements}

In addition to the direct effects of egg mortality on recruitment rates to a population, there are indirect effects of adult female mortality that influence recruitment potential. Adult female copepods do not release fertilized eggs immediately upon the terminal molt but must first complete oogenesis and must be inseminated. Also, under some circumstances females can survive to a post-reproductive or 'spent' stage. Adult females in these different reproductive states have unequal probabilities of egg release, resulting in per capita fecundity below that expected from the maximum obtained from reproductively viable females in the laboratory. The mortality rate influences the proportions of females in these different states (OHмAN \& al. 1996). If the mortality of adult females varies substantially over time, the proportion of all adult females that are in the 
reproductively active stage will shift greatly. Mortality variations of adult females can create at least 2-4 fold variations in per capita egg production over time (OHMAN $\&$ al. 1996). The causes for this variability in per capita egg production rates are likely to be misinterpreted and assigned exclusively to food limitation unless mortality is accounted for. However, if the total population of females is always sampled in a random manner, then measurements of per capita fecundity should be unbiased even if the causes of variations in fecundity are not well understood.

\section{DEVELOPMENT RATE, STAGE DURATIONS AND GENERATION TIMES}

Copepods do not carry definite, countable indications of individual age comparable to otolith layers in fish. Thus, age composition of a sample can only be approximated. Life cycles of copepods are divided into discrete, distinguishable stages so that stage composition can sometimes serve as a proxy for age composition. Free-living copepods usually have 13 developmental stages. Most other zooplankton taxa, even other crustaceans, are less discrete and rigid in development pattern so that a relation between size and age must be developed to approximate age composition.

Copepods have been successfully reared for over 25 years, since the first successes of ZiLlioux \& Wilson (1966) with Acartia, Muldin \& Brooks (1967) with Calanus and Rhincalanus, and CoRKeTt (1967) with Pseudocalanus. Laboratory rearing makes possible estimates of stage duration and, thus, development rate. The problem which must be overcome is that copepods have substantial variance among individuals in development rate, so that useful estimates of central tendency require data from many individuals. Two techniques have been applied. The most obvious is to rear individuals in separate containers, checking each at intervals and recording the times of successive molts. Because good precision requires frequent observations (which to date have not been readily automated) night and day for many days, this has rarely been applied. It also forces modest sample sizes. However, it allows study of how variance in developmental timing is structured. For example, Carlotti \& Nival (1992) showed for Centropages typicus that individuals spending relatively long (or short) intervals in one stage tend to have long (or short) intervals in other stages as well.

The other technique is to rear a large group of individuals together, starting them in rough synchrony as eggs or sorted early stages. As development progresses, this group is sampled at suitable intervals and the proportions in different stages determined by counting. Cumulative proportions are then plotted in a form apparently developed by Heinle (1966). Functions are fitted to the time sequences of cumulative proportions of each stage, providing some smoothing of the proportion estimates from samples of modest size. KLEIN BRETeler \& al. (1994) have suggested a probability density function based on the gamma distribution for this purpose and have provided a fitting technique. Linear regressions have been used in a number of studies, and serve reasonably well, since it is mainly the position of the median crossing that is of concern. Estimates of median stage durations are taken as the time intervals between crossings of the $50 \%$ line by successive stage functions (see the 'Cohort method' and Eq. 16). While this technique has been repeatedly applied (see PETERSON \& Painting 1990; Klein Breteler \& al. 1994 for references), its statistical properties need further study. It is possible, in circumstances where reproduction occurs over a limited time interval and advection is limited, to apply the cumulative proportion technique to changing copepod stage proportions observed in the field (e.g. Miller \& Nielsen 1988; Miller 1993). However, differences in mortality rates between stages produce changes in stage proportions additional to those resulting from progression in development. Models (Miller 1993; Miller \& Tande 1993) suggest that the impact of differential mortality is modest, so that such stage duration estimates still have value.

A field approach to estimation of copepod stage duration was suggested by BurKILl \& Kendall (1982) and modified by KimMerer \& McKinNon (1987). In simplest form a live sample is collected from the field and a stage of interest is sorted from it into one or several containers. Then over a suitable period one determines the proportion of individuals that have molted to the next stage. Assuming steady state recruitment to the stage, trivial mortality and no change in development induced by experimental conditions (all frequently unrealistic), the inverse of the rate of increase of proportion molted should be the stage duration (MILLER \& al. 1984). KiMMERER \& MCKINNON (1987) modified this by working with more than one stage. Mixed copepodites are collected by gentle net tow, sieved twice to remove the oldest stages (retained by a coarse mesh) and the youngest stages (passing a finer mesh), then resuspended and held for an interval to allow development. Stage proportions of initial and final samples are determined and molting rates for each stage are estimated (PETERSON $\&$ al. 1991) from:

$$
m_{i}=\frac{\sum_{k=i+1}^{q} p_{k}\left(t_{2}\right)-\sum_{k=i+1}^{q} p_{k}\left(t_{1}\right)}{p_{i}\left(t_{1}\right)\left(t_{2}-t_{1}\right)}
$$

where $\Sigma p_{k}\left(t_{2}\right)$ and $\Sigma p_{k}\left(t_{1}\right)$ are the estimated final and 
starting proportions of stages older than the stage of interest, and $p_{i}\left(t_{1}\right)$ is the estimated starting proportion of that stage. From equations elaborated later on (Eqs $11 \mathrm{a}, \mathrm{c})$ it can be shown that the stage duration $\left(a_{i}\right)$ can be found from $m_{i}$ according to:

$$
a_{i}=\frac{\ln \left(\frac{d_{i}}{m_{i}}+1\right)}{d_{i}}
$$

if it is assumed that the recruitment to stage $i$ is constant during the last $a_{i}$ days before sampling the individuals. Note that the death rate in Eq. $8 \mathrm{~b}$ refers to the death rate of the natural population (i.e. the death rate that forms the age structure within stage of the sampled individuals) and not the death rate during incubation. In the case where the natural death rate is zero, Eq. $8 \mathrm{~b}$ reduces to

$$
a_{i}=m_{i}^{-1}
$$

Once stage duration or development rate data are obtained, it is valuable to model them as functions of controlling variables. On the whole the dominant controlling variable is temperature, with slowing effects from food when it is in short supply. Arguments have been advanced (e.g. HunTLEY \& LOPEZ 1992) that food is rarely in short supply, at least in respect to requirements for development. Therefore, models of the response to temperature alone are often used. One should, however, be aware of the bias this may introduce. Models might be given preference as deriving from first principles or as convenient for use in practice. On the whole, the latter basis prevails. MCLAREN (1963) suggested and carefully justified a convenient functional form due to BELEHRÁDEK (1935), and it has been widely applied. In the Belehrádek formulation stage duration (or development time), $D$, is given by

$$
D=a(T-\alpha)^{b}
$$

where $\mathrm{T}$ is temperature (Celsius) and $a, \alpha$ and $b$ are fitted parameters. McLaren showed that $a$ corresponds to differences in mean slope, $\alpha$ to position along the temperature scale and $b$ to curvilinearity. Fitted to appropriate data, this function (or other suitable ones) can be used in detailed analyses in field situations with variable temperature. However, the available data are sparse.

Estimation of average stage durations can be difficult if a development stage is distributed over a broad range of depths in a water column that is temperature-stratified. Animals of the same stage that develop at different rates may occur in different strata. Fine-scale vertical sampling and incubations to determine a distribution of development rates at different temperatures may help address this problem.

\section{MORTALITY ESTIMATION DURING INTERVALS WITH NO RECRUITMENT}

The life history of some zooplankton species is characterized by extended periods without recruitment to one or more development stages. Such periods, which may be indicated by the absence of mature females and/or by zero-counts for one or more of the younger development stages (or size groups), enable almost direct estimation of mortality. For example if the egg, naupliar stages and the three first copepodite stages are absent, because of ceased recruitment, changes in the total numbers (i.e. the combined stage CIV-CVI) during the period without recruitment is due to mortality only (if advective and migratory influence can be ruled out). Hence, the per capita mortality rate for the stages present can be found by the relationship (or by a fitting procedure applied on a series of $n$-estimates):

$$
d_{i . . q}=\frac{\ln \left[n_{i . q}\left(t_{1}\right)\right]-\ln \left[n_{i . q}\left(t_{2}\right)\right]}{t_{2}-t_{1}}
$$

where $n_{i . q}(t)$ is the total abundance of the combined stages $i+1 \ldots q$ at time t. Although if recruitment to a particular stage has ceased, note that this expression should not be applied to a single stage where individuals still mature to the next stage. Then a combination with the older stages, as indicated by Eq. 10, is required. This estimation procedure should be considered robust as it does not depend on either recruitment estimates or development times, but only on the precision of the abundance estimates. Although timedependent mortality rates can be obtained by this procedure, stage specific mortality estimates require parameterization of recruitment and maturation of the individual stages (see next section). Eq. 10 should be considered in mortality estimation when recruitment to one or more of the younger stages has ceased. The population development of Calanus hyperboreus (Matthews \& al. 1978) and Calanus finmarchicus (AKSNES \& MAGNESEN 1983) accomodated this approach. In the following section we describe approaches for mortality estimation for populations with simultaneous recruitment and mortality. 


\section{PARAMETER ESTIMATION FROM CENSUS DATA}

Estimation is the inference of population parameters or quantitative process rates that cannot be observed directly or are difficult to observe directly. These inferences are made from observations of changes in population abundance over time. In practice the abundance observations must usually be structured by age or some anatomical traits monotonically linked to age. The quantities that are to be estimated include mortality rate, birth rate and development rate, but the fewer of these that have to be estimated simultaneously the better. For copepods the population data will usually consist of abundance estimates for a series of development stages, at a series of sampling times. For example, population data might be available for eggs, naupliar stages I-VI, copepodite stages I-V, and adults. In general, the better sampling coverage of the different life cycle stages the better the estimates will be.

Reviewing the population dynamics literature gives a first impression that it provides more estimation techniques than estimates. At closer inspection, however, most techniques are closely related. The differences are best described in terms of the assumptions of the models underlying the techniques. In the following discussion we will concentrate on the assumptions which provide a reasonable framework for classification. The vertical approach utilises the stage-structure of samples taken at the same point in time. This introduces assumptions about constancy in the parameters but relaxes assumptions concerning advective influence. The horizontal approaches utilize both stage structure within samples as well as temporal changes in absolute abundances. In this way the assumption about constancy in the population parameters can be relaxed, but the requirement of lack of advective influence is introduced. Of the horizontal methods, different variants of what can be commonly termed cohort methods have been widely used. These methods provide mathematical simplicity, but are based on more restrictive assumptions than the methods fitting predescribed models of population dynamics to census data.

\section{THE VERTICAL LIFE TABLE APPROACH}

The basic equation of the vertical approach describes the number observed in a development stage $\left(n_{i}\right)$ at a point in time $\left(t_{j}\right)$ as a function of recruitment, mortality and stage duration:

$$
n_{i}\left(t_{j}\right)=\int_{t_{j}-a_{i}}^{t_{j}} R_{i} e^{-d_{i}\left(t_{j}-t\right)} \mathrm{d} t=R_{i}\left(1-e^{-d_{i} a_{i}}\right) / d_{i}
$$

(a juvenile stage)

$$
n_{q}\left(t_{j}\right)=\int_{-\infty}^{t_{j}} R_{q} e^{-d_{q}\left(t_{j}-t\right)} \mathrm{d} t=R_{q} / d_{q}
$$

(the adult stage)

The recruitments of consecutive stages is connected by the equation:

$$
R_{i+1}=R_{i} e^{-d_{i} a_{i}}
$$

It is assumed that the recruitment to the stage, the stage duration, and the mortality for a stage are constant (actually, Eqs 11a and b are also derived by introducing these assumptions into Eq. 5 and setting $\mathrm{d} n / \mathrm{d} t=0$ ). Note, however, that for the juvenile stages these assumed constancies are required only for a period of time that corresponds to the duration of the stage. In the approach originally formulated for zooplankton by MULLIN \& BROOKS (1970) and further elaborated by FAGER (1973) and AKsnes \& OHMAN (1996) a common mortality rate for two consecutive stages may be calculated according to:

$$
\begin{gathered}
\frac{e^{d_{i} a_{i}}-1}{1-e^{-d_{i} a_{i+1}}}=f_{i} \quad \text { (two juvenile stages) } \\
d_{q-1}=\frac{\ln \left(f_{q-1}+1\right)}{a_{q-1}} \quad \text { (12b) } \\
\text { (juvenile and adult stage) }
\end{gathered}
$$

where $d_{i+1}=d_{i}$ (and $d_{q}=d_{q-1}$ ) is the common mortality rate for the two stages analysed, $\mathrm{f}_{i}=\mathrm{n}_{i} / \mathrm{n}_{i+1}$ where $\mathrm{n}_{i}$ is the number of individuals in development stage $i$, and $q$ denotes the final stage in which individuals are leaving by dying only. As demonstrated by simulation (AKSNES \& OHMAN 1996) mortality estimates obtained by this method are reliable only if the estimate is based on a large number of samples (typically more than 10) such that the values of $f_{i}$ are not biased by small-scale patchiness. The method provides accurate mortality estimates for variable recruitment as long as this variability can be considered random. In the special and unusual case of isochronal development (i.e. $a=a_{i}=$ $\left.a_{i+1}\right)$, Eq. 12a reduces to $d_{i}=\ln \left(f_{i}\right) / a$ which corresponds to the relationship used in the vertical approaches of Heinle (1966), Durbin \& Durbin (1981) and Kimmerer \& MCKInNon (1987). Similarly, by assuming zero mortality we obtain the relationship used by UyE (1982) in 
estimation of stage-specific daily recruitment rate; $R_{i+1}=n_{i} / a_{i}$ (an expression suggested by EDMONDSON \& WINBERG 1971). Still another version of the vertical approach was used by KıøRBOE \& al. (1988) and KiøRBOE \& NIELSEN (1994) in their estimation of egg mortality $\left(d_{1}\right)$ :

$$
d_{1}=\frac{b_{f} n_{f}}{n_{1}}-m_{1}
$$

where $b_{f} n_{f}$ is the measured total egg production rate $\left(b_{f}\right.$; individual female egg-laying rate, and $n_{f}$; number of females) and $m_{1}$ is the hatching rate. Zero mortality is assumed in the hatching term if $m_{1}$ is taken as the inverse of the egg duration time $\left(m_{1}=a_{1}^{-1}\right.$, KiøRBOE $\&$ al. 1988). In the case of non-zero mortality (see Eq. 8b); $m_{1}=d_{1} /\left(\exp \left(d_{1} a_{1}\right)-1\right)$ should be inserted into Eq. 13:

$$
\frac{\left(1-e^{-d_{1} a_{1}}\right)}{d_{1}}=\frac{n_{1}}{b_{f} n_{f}}
$$

which is identical to eq. 5 of Peterson \& Kimmerer (1994) used in the estimation of egg mortality in Temora longicornis. (Note, however, that their equations 4 and 5 have a typographical error in the localization of the brackets).

The main assumption underlying the vertical approach is that daily recruitment to a particular stage should not be characterized by an upward or downward trend over a time period corresponding to the duration of two consecutive developmental stages. In order to improve the accuracy of the mortality estimates, knowledge about short-term trends in recruitment can be accounted for by inclusion of the rate of change in the recruitment rate in the estimation model (AKsnes \& Ohman 1996). The strength of the vertical approach is that different samples do not need to represent a constant fraction of the spatial volume occupied by a homogenous population such as required in the horizontal time-series approaches. The advective problem is eliminated as long as the individuals in a sample have been influenced equally by advection (the horizontal approach requires unbiased estimates of the absolute population abundance). Furthermore, $f_{i}$-estimates are generally less variable than $n_{i}$ estimates due to the tendency for co-variance of abundances of different development stages. This fortunate property is probably due to the common advective regime experienced by the animals of a single sample. Individuals of different samples (temporal and spatial) are much more likely to have different advective histories.
A disadvantage of the vertical method is that it provides mortality estimates for pairwise development stages, rather than for individual stages, and that the assumption about constancy in recruitment will often be inappropriate. In addition the method should not be applied in regions with strong spatial gradients of predation, feeding opportunities, temperature, or other factors causing pronounced spatial variations in development times and mortality rates. Generally, the vertical approach should be used primarily when the principle assumptions are met and horizontal techniques are likely to fail due to advective influence or due to the lack of adequate time-series.

\section{THE HORIZONTAL LIFE TABLE APPROACH}

\section{The cohort method}

We do not consider the cohort method as a standarized technique, but rather a term used to characterise a collection of different techniques with a similar approach. Frequently, quantification of stage durations, total recruitment (and not the recruitment rate) and/or survivorship has been the aim of applying such techniques. The method requires that discrete cohorts can be identified and sampled throughout their development. It is generally assumed that all individuals of a particular stage of a cohort can be characterized by a common stage duration and mortality. As discussed below, however, the assumption about the nature of the mortality has often been more restricted. In the cohort approach, the population parameters are commonly related to areas, temporal means and medians of the stage frequency curves in relation to a specified model. Estimation of these statistical properties of the stage frequency curve provide estimates of the population parameters. As with the vertical methods, the assumptions of the underlying estimation model of different cohort analyses are often unspecified or even mis-specified, and the differences between approaches are often found in hidden assumptions. In the next section, we will make some clarifications and distinguish between those estimation models that assume no mortality (in practice negligible mortality) and those that include mortality.

Estimation models assuming negligible mortality. In an extensive investigation of Acartia clausii (=hudsonica) LANDRY (1978) applied the method of RigLER \& COOLEY (1974). Recently this method was also applied by Peterson \& Kimmerer (1994) in their study of Temora longicornis. In this method the total recruitment $\left(T_{i}\right)$ to and duration $\left(a_{i}\right)$ of a developmental stage $(i)$ are obtained according to: 


$$
\begin{gathered}
T_{i}=\frac{A_{i}}{a_{i}} \\
\frac{1}{2}\left(a_{i+1}+a_{i}\right)=B_{i+1}-B_{i}
\end{gathered}
$$

where $A_{i}$ and $B_{i}$ are the calculated area and temporal mean of the stage frequency curve. In order to estimate stage durations Eq. $15 \mathrm{~b}$ requires that the duration of at least one developmental stage is known independently. Estimates of stage specific survival $\left(S_{i}\right)$ and instantaneous mortality rate $\left(d_{i}\right)$ have frequently been obtained by the relationships $S_{i}=T_{i+1} / T_{i}$ and $d_{i}=-\ln \left(S_{i}\right) a_{i}$. UYE (1982) used a similar method based on GeHrs \& Robertson (1975) in the construction of life tables for Acartia clausii. GeHrs \& ROBERTSEN (1975) provided a formula for estimation of recruitment that is identical to Eq. $15 \mathrm{a}$.

It has frequently been asserted that Eqs 15 and 16 are valid if the mortality rate is the same for all development stages analysed. As discussed by HaIRSTON \& Twombly (1985) and AksNes \& HøisÆTER (1987), however, Eqs $15 \mathrm{a}$ and $15 \mathrm{~b}$ are valid only when instantaneous mortality rate is zero, and become increasingly biased with increased mortality. Hence, Eqs $15 \mathrm{a}$ and $15 \mathrm{~b}$ are primarily appropriate for field data without significant mortality, but should generally not be applied for populations experiencing moderate or high levels of mortality.

Estimation models including mortality. A method that is related to Eq. $15 \mathrm{~b}$ has become popular in the estimation of stage durations. Here, stage duration is related to the difference in medians, rather than to the difference in temporal means (see Peterson \& Painting 1990 and KLEIN BRETELER \& al. 1994 for review). In this method, the median $\left(H_{i}\right)$ is defined as the time when $50 \%$ of the individuals of an entire cohort is observed in stage $i$ or later stages and the other $50 \%$ is occupying the earlier stages. The stage duration is then calculated by the difference in successive medians:

$$
a_{i}=H_{i+1}-H_{i}
$$

As discussed previously different methods have been applied to increase the precision of the $H$-estimates. As demonstrated by Miller (1993) and Miller \& TANDE (1993) the accuracy of this method is not affected by mortality per se, but is sensitive to differentials in mortality between stages. However, their study shows that the bias is mild and that the method (denoted the "Heinle graph' method) gives useful estimates of stage durations. As advocated by AKSNES \& HøISÆTER (1987) the fol- lowing equations (originally derived by MANLY 1977) are appropriate for the situation where mortality is acting on the cohort.

$$
\begin{array}{cc}
A_{i}=\frac{T_{i}\left(1-S_{i}\right)}{d_{i}} & \text { (juvenile stage) } \\
B_{i}=\mu_{i}+\frac{1}{d_{i}}-\frac{a_{i} S_{i}}{1-S_{i}} & \text { (juvenile stage) } \\
A_{q}=\frac{T_{q}}{d_{q}} & \text { (adult stage) } \\
B_{q}=\mu_{q}+\frac{1}{d_{q}} & \text { (adult stage) }
\end{array}
$$

and the equations connecting the stages;

$$
\begin{gathered}
T_{i+1}=T_{i} S_{i} \\
\mu_{i+1}=\mu_{i}+a
\end{gathered}
$$

where stage specific survival is $S_{i}=\exp \left(-d_{i} a_{i}\right)$, and $\mu_{i}$ is a parameter describing the time for peak recruitment to the stage. Eqs $17 \mathrm{a}$ and $17 \mathrm{~b}$ correspond to Eqs $15 \mathrm{a}$ and $15 \mathrm{~b}$, but account for influence of mortality. With these more general equations it can be seen how the area $\left(A_{i}\right)$ and the temporal mean $\left(B_{i}\right)$ of a stage frequency curve are affected by the mortality as well as by the recruitment and stage duration. Note also that the equations simplify in the case where all individuals leave the stage by dying (such as the adult, the total abundance, or any other combination of stages that include the adult stage). Rather than one estimation technique, Eqs 17a-f should be regarded as a set of equations that connects the population parameters to the first (area) and second moment (temporal mean) of a complete stage frequency curve. Inclusion of independent knowledge of the stage durations and/or recruitment parameters, combination of stages, assumption of equiproportional development, and introduction of the assumption that mortality rate is equal for some or all stages represent different modifications to the technique (MANLY 1977; AKSNes \& Magnesen 1983, 1988; Manly 1990; Wood 1994). An important drawback associated with cohort methods is that no error measures of the parameters are obtained unless some kind of jack-knife technique for variance estimation is applied (MANLY 1977).

Requirement of complete temporal sampling coverage. The accuracy of any cohort method depends upon sampling the entire curve of abundance versus time (see 
MANLY 1985 and 1990, however, for description of an iterative method that compensates for inadequate sampling). Particularly, the $B$-estimates (which may be viewed as a measure of the 'center of gravity' ) can become seriously biased when part of the abundance curve is missing. Each stage included in an analysis should be covered by sampling from the date the first individual enters the stage until the date the last individual leaves. Otherwise, the accuracy of the cohort method is likely to be poor.

\section{General estimation techniques}

Now we will consider the most general techniques that have been applied in extracting demographic parameters of zooplankton populations. The estimation process has two main parts: a model of the population under study must be formulated and this must then be fitted to the observed data by some means. Consideration must be given to the need to obtain uncertainty estimates for all estimated quantities and to the fact that the correct structure for the fitted model is rarely known exactly, something which must be included in uncertainty estimates if they are to have validity.

Models. If the birth, death, immigration and emigration rates to a population are all known at any time, along with some initial population size, then it is a matter of straightforward arithmetic to work out the population size at any time: no model is required. Unfortunately even with perfect data this is very rarely the case if we attempt the reverse calculation, and try to calculate birth, death and migration rates from population data. In this latter circumstance, there will always be an infinite number of combinations of underlying population rates, which could have produced the observable changes in population size (for example increased recruitment to a population is usually indistinguishable from decreased death rate). Thus we must formulate a model of the population whose hidden process rates (births, deaths etc.) we wish to find: the model serves to codify our prior beliefs about the population under study and thus to select the most plausible combinations of birth and death rates, which are consistent with our data, given those prior beliefs. The methods presented so far have all implicitly involved models of the population under study: this section deals more generally with the process of explicitly specifying an appropriate model and fitting it to the data.

A good model is crucial to the success of attempts to extract population parameters from population timeseries, and getting the right model can be the hardest part of any study. A model that is very general will not reduce the range of parameter values consistent with the data, so that estimates will have high variance but low bias. A more restrictive model is desirable, but only if the model is based on concrete knowledge. Without such knowledge a model may be mis-specified and parameter estimates will be biased, by an unknown (but possibly substantial) amount. Arguably, this is worse than having estimates with high variance but low bias, as the latter at least represent fairly what is really known. In summary: models used for parameter estimation should not be less general than is justified by actual knowledge.

There are a number of general types of model that provide good frameworks for building models of zooplankton population dynamics. They fall into 3 main categories: delay differential equation models (GURNEY $\&$ al. 1986), partial differential equation models (e.g. Metz \& Diekmann 1986), and matrix population models (see CASWELl 1989).

Delay differential equation models describe the discrete stage populations as continuous functions of time. An appropriate general model of this type (again treating stages as age classes) has already been discussed (see Eqs 5a-d). These models require initial continuous age (or development) structures within each stage as boundary conditions. Examples of estimation methods based on these models are 'systems identification' approaches (PARSLOW \& al. 1979; SonNtag \& Parslow 1981; HAY \& al. 1988). In a closely related approach suggested by MANLY (1974) and applied by Matthews \& al. (1978), Parslow \& al. (1979) and Aksnes \& MAGNeSEn (1983) a pre-described recruitment function is specified and the number in a stage as a function of time is explicitly represented by the model and fitted to the observations.

Partial differential equation models (PDE) treat time and age (or growth index) as continuous variables. An example of a PDE model which may be appropriate for zooplankton populations is the McKendrick-von Foerster equation (here defined in terms of age):

$$
\frac{\partial n}{\partial t}+\frac{\partial n}{\partial x}+d(x, t) n(x, t)=0
$$

Here $n(x, t)$ is the number of individuals (per unit age interval) of age $x$ at time $t$, and $d(x, t)$ is the per capita death rate of individuals of age $x$ at time $t$. In general such a model requires specification of an initial age structure $n\left(x, t_{0}\right)$ and recruitment rate $n(0, t)$ although the latter may be related to the adult population. The abundances of particular stages $\left(n_{i}(t)\right)$ are related to $n(x, t)$ as follows: if the individuals in stage $i$ at time $t$ lie between the ages of $\alpha_{i}$ and $\alpha_{i+1}$ and (where the stage duration is $a_{i}=\alpha_{i+1}$ $-\alpha_{i}$ ) then we have that 


$$
n_{i}(t)=\int_{\alpha_{i}}^{\alpha_{i+1}} n(x, t) d x
$$

Clearly, the quantities $d(x, t), \mathrm{n}\left(x, t_{0}\right), \mathrm{n}(0, t)$ and $\alpha_{i}$ are all candidates for estimation, given some set of observations of the stage populations. Examples of estimation methods based on these models are Wood \& NisBeT (1991), Wood (1994), BANKs \& al. (1991) and OHMAN \& Wood (1996). In the case where stage durations are assumed known a priori, the simulation study by WOOD (1994) demonstrated that this approach provided more accurate estimates than the 'cohort method', the 'projection matrix method', and the 'systems identification method'. Although results from such comparison should be interpreted with care, since methods are sensitive to violation of different assumptions, the 'population surface method' of Wood (1994) is promising. The method makes few assumptions about the nature of the population dynamics. The most important is, as with most other methods, that there is no variability in stage duration between individuals at a particular time, although this assumption is easy to relax if the distribution of development times is known.

The final set of models are the matrix models where a matrix projects a vector of stage abundances at one time to the vector of stage abundances some fixed time later. An appropriate general form for zooplankton populations is an equation of the form:

$$
\mathbf{n}\left(t_{j+1}\right)=\left[\begin{array}{cccccc}
P_{1} & 0 & \cdot & \cdot & \cdot & \beta \\
G_{1} & P_{2} & \cdot & \cdot & \cdot & 0 \\
0 & G_{2} & P_{3} & 0 & \cdot & 0 \\
\cdot & \cdot & \cdot & \cdot & \cdot & \cdot \\
\cdot & \cdot & \cdot & \cdot & \cdot & \cdot \\
\cdot & \cdot & \cdot & \cdot & G_{q-1} & P_{q}
\end{array}\right] \mathbf{n}\left(t_{j}\right)
$$

where the vector $\mathbf{n}\left(t_{j}\right)$ is a vector containing the abundances of the stages at time $t_{j}$, and the matrix elements are as follows: $G_{\mathrm{i}}$ is the proportion of the individuals in stage $i$ at $t_{\mathrm{j}}$ that will have matured to stage $i+1$ and still be alive by time $t_{j+1} ; P_{\mathrm{i}}$ is the proportion of individuals in stage $i$ that will survive, but still be in stage $i$ by $t_{j+1}, \beta$ is the net reproductive rate of individuals in the final (adult) stage. Note that it is possible to include further terms in the transition matrix if individuals can move to stages other than the next one, during one time step, and that further birth rate terms can be added if individuals can reproduce before the last stage. The parameters of the transition matrix may be time dependent. We would like to estimate the parameters of the transition matrix given estimates of the stage abundances. Examples of estimation methods based on matrix models can be found in CASWELL \& Twombly (1989) and Twombly (1994). An advantage of this model is that the stage duration is not assumed to be fixed among individuals such as in the other models. The probabilistic representation, however, requires frequent sampling unless more non-zero transition-parameters are entered the projection matrix. Generally, such increases in the number of parameters to be estimated (or to be known independently) is a problem.

The main difference between the above models and the mathematically less sophisticated models underlying the vertical approach and the cohort methods is the level of generality. The biological assumptions of the simpler methods are more restrictive. The general models can be used as the starting point for developing specific models of a particular system. Functional forms are assumed for the various population rates within the models, and the parameters of these assumed functions can be found by fitting the model to data (see next section). Alternatively, the population rates can be described in a very general way, making only limited assumptions about functional form using spline functions. These are functions defined as the smoothest curves through a set of points. The set of points then defines the curve uniquely, and points can be treated as free parameters: with enough such points almost any smooth function can be represented to arbitrary accuracy. When fitting models involving spline functions, it is easy to include penalty functions which enable the effective complexity of the spline functions to be controlled. As a concrete example, we might wish to represent per capita death rate as a smooth function of time. If we choose to do so with a spline having 10 parameters, then the complexity of the death rate function can be varied from a straight line to the complexity of a 10th order polynomial, by varying a single parameter. It is then possible to use the population data themselves to select the optimal amount of complexity for the death rate function, and also to estimate the degree of error in parameter estimates due to use of the wrong form of the death rate function. For examples of the application of these ideas see Wood \& Nisbet (1991), Wood (1994), OHMAN \& Wood $(1995,1996)$.

Models may need to be modified for species whose life history includes an interval of dormancy. Where benthic resting eggs are produced or late developmental stages enter diapause deep in the water column (e.g., copepodid IV or V of some members of the Calanidae), both the recruitment rate and mortality rate are likely to change. If one fraction of the population enters dormancy concurrently with the continuous development of another fraction, this can be modelled by describing individuals entering dormancy as a separate stage from those exhibiting subitaneous development. Care must 
also be taken to differentiate these stages in field samples.

Two important issues must be considered when formulating models. The first is that if the model is too sparse, making too many assumptions about the nature of the populations dynamics, then the component of the error in estimates due to model mis-specification will be high, but hard to quantify. Unfortunately, if the model is very complicated and detailed with lots of parameters, then the parameter estimates are likely to be very poor unless a perfectly correct model is fitted to absolutely precise and accurate data. In addition, even quite simple models can fall victim to unpleasant instabilities in estimation of their parameters. This is usually caused by over-estimation of mortality rate in one stage being almost exactly compensated for by underestimation of mortality rate in the next stage (this also happens when fitting stage durations); see Wood \& al. (1989) or Wood \& NisBet (1991) for details. This problem can be combated if a model can be formulated which requires slow changes in mortality rate through the stages in the population. This type of restriction can be incorporated either by careful formulation of a parametric model, or by incorporating a penalty for rapid variation in rates from one stage to the next. A problem similar to the mortality rate instability is that an overestimation of development time can result in an underestimation of mortality rate.

The second issue to consider is actually helpful to the estimation process. Many population rates are intrinsically positive. Death rate and birth rates cannot be negative and growth rates can often be assumed not to be. These facts place restrictions on the range of allowable parameters when fitting a model-i.e. they reduce the number of ways in which any set of observed population data could have been produced. Again the restrictions can either be incorporated implicitly in a carefully formulated model, or they can be imposed as restrictions when the model is fitted to data. Note, however, that advective processes can generate negative birth and death rates when a population model not including advection is applied.

Fitting models. Broadly there are two approaches to model fitting. The first is the regression type approach, where the model is viewed as a method for predicting the stage abundances at one sample time on the basis of the stage populations at an earlier time. This type of approach is most useful for matrix models. The second approach is more conventional model fitting in which the parameters are sought which allow the model to best fit the stage frequency data.

The simplest least squares problems occur when the model is linear in its parameters, and the model can be written as:

$$
\mathbf{n}^{\mathrm{T}}=\mathbf{L} \mathbf{p}
$$

where $\mathbf{n}^{\mathrm{T}}=\left[n_{1}\left(t_{1}\right), n_{2}\left(t_{1}\right), \ldots . ., n_{q}\left(t_{1}\right), n_{1}\left(t_{2}\right), n_{2}\left(t_{2}\right), \ldots \ldots\right.$, $\left.n_{q}\left(t_{2}\right), \ldots . ..\right]$ is a vector containing all the modelled stage abundances at each sampling time, $\mathbf{p}$ is a parameter vector and $\mathbf{L}$ is a matrix of coefficients (which will actually be stage abundance values for regression methods). Usually we have a vector of observed abundances $\mathbf{Y}$ corresponding to $\mathbf{n}$, and we would like to find the parameter vector $\mathbf{p}$, which minimises the sum of squares of deviations of model from data, that is:

$$
\begin{gathered}
(\mathbf{Y}-\mathbf{n})^{\mathrm{T}}(\mathbf{Y}-\mathbf{n})=(\mathbf{Y}-\mathbf{L} \mathbf{p})^{\mathrm{T}}(\mathbf{Y}-\mathbf{L} \mathbf{p}) \\
(\mathbf{Y}-\mathbf{L} \mathbf{p})^{\mathrm{T}}(\mathbf{Y}-\mathbf{L} \mathbf{p})=\mathbf{p}^{\mathrm{T}} \mathbf{L}^{\mathrm{T}} \mathbf{L} \mathbf{p}-2 \mathbf{p}^{\mathrm{T}} \mathbf{L}^{\mathrm{T}} \mathbf{y}+\mathbf{y}^{\mathrm{T}} \mathbf{y}
\end{gathered}
$$

This is minimised when $\mathbf{p}=\left(\mathbf{L}^{\mathrm{T}} \mathbf{L}\right)^{-1} \mathbf{L}^{\mathrm{T}} \mathbf{y}$. The standard estimate of the covariance matrix for the parameters is $\operatorname{Cov}(\mathbf{p})=\left(\mathbf{L}^{\mathrm{T}} \mathbf{L}\right) \hat{\sigma}^{2}$ where $\hat{\sigma}^{2}(\mathbf{n}-\mathbf{Y})^{\mathrm{T}}(\mathbf{n}-\mathbf{Y}) / n$ and $n$ is the number of data points less the number of parameters. Notice however that this covariance matrix is only valid if the model is the correct description of the population.

Commonly, models are not linear combinations of parameters, but non-linear functions of parameters, hence non-linear least squares methods must be used to perform fitting. The objective is still to find the parameters of the model which minimize the sum of squares of deviations of model from data, but this requires iterative methods. A good choice is the Levenberg-Marquardt method, which combines steepest descent minimisation with a quadratic model of the least squares objective function, to produce a very robust and quite efficient model fitting method. Details can be found in PrEss \& al. (1988). In addition most of the major numerical software libraries provide routines for non-linear least squares fitting.

As mentioned in the model section it is often desirable to constrain the parameters of a model in order to preserve biological realism in the form of non-negative population death rates and birth rates. If the model has been set up so that these constraints can be expressed as linear constraints on the parameters of the model (i.e. can be written in the general form $\mathbf{A p} \geq \mathbf{b}$ where $\mathbf{A}$ and $\mathbf{b}$ are a coefficient matrix and coefficient vector respectively) then methods exist for solving the least squares problems subject to the constraints. For linear problems the method is known as quadratic programming, and for non-linear problems there are iterative methods based on successive approximation by quadratic programming problems. Details are given in GILL \& al. (1981) and again the major numerical software libraries have 
routines for solving constrained least squares problems.

When fitting models with constraints on how rapidly population rates are allowed to vary, it is necessary to have some method to choose how strongly such constraints should be applied. This can be done by the method of cross-validation, which is equivalent to seeing how well on average a model fits data which were not used in calculating the model, and choosing the model which does best by this criterion. In this case the different models differ in the strength of the constraints on how rapidly their parameters should vary. The cross validation approach leads naturally into a means of dealing with uncertainty about what model should best be used. Details are given in Wood (1994).

Matrix regression methods can easily be implemented in various packages allowing matrix manipulation (for example MATLAB). It is fairly straightforward to fit delay differential equation models using the routines given in PRESS \& al. (1988) or using routines from NAG or IMSL.

\section{RECOMMENDATIONS}

Unfortunately, the diversity of copepods life histories and the nature of the marine environment make it unwise to suggest one highly standarized protocol for population dynamics investigations. Accordingly, our recommendations are formulated at a rather general level:

- If possible, accurate development times and egg production rates (recruitment) should be measured experimentally. This reduces the number of parameters to be estimated simultaneously from census data. The best situation is when mortality rate is the only parameter to be estimated from the census data.

- Periods without recruitment to one or more development stages permit almost direct measurements of mortality (Eq. 10). Such periods are indicated by the absence of mature individuals and/or by zero counts for one or more of the younger development stages (or size groups).

- Vertical distribution and migration are important aspects of zooplankton population dynamics. Surface integrated sampling may give rise to biased stage composition. Broad depth distributions in a temperature stratified water column may generate broadly distributed stage durations. Estimation techniques are extremely sensitive to such biases which should be given particular attention in zooplankton studies. Thus assessment of the vertical distribution and behavior should be an integrated part of studies on zooplankton population dynamics.
- Choice of estimation technique should be governed by: 1 . How closely the model underlying the method matches your population. The greater the violation of the model assumptions by the study population the greater unknown bias in parameter estimates will be. 2 . Whether the sampling error distribution assumed by the method is reasonable for the data in hand. 3. Whether the method allows you to estimate reliable confidence intervals for inferred quantities and to estimate the effect of model mis-specification. 4. If migrations (including advective influence) can be ruled out as influencing the observed dynamics, parameter values such as death rate, birth rate and population size should be constrained so they are positive during the estimation process.

- If advection influences the dynamics (Eqs 1-4 may provide guidance) or we do not have adequate timeseries for the horizontal methods, the vertical life table approach should be considered in mortality estimation. Note that this method requires a large number of samples closely spaced in time. On the other hand, if advective influence is known to be low and adequate time series are available horizontal methods should be applied.

- Simulations give valuable guidance to studies of population dynamics in general, and more specifically to tests of different estimation techniques under specified conditions. If an estimation technique doesn't work on simulated data it will certainly not work on real data.

\section{ACKNOWLEDGEMENTS}

We thank two anonymous reviewers for valuable comments on the manuscript. This work has been supported by grants from the Research Council of Norway to D.L. Aksnes and by NSF OCE 9613596 to M.D. Ohman.

\section{REFERENCES}

Aksnes, D.L. 1996. Natural mortality, fecundity and development time in marine planktonic copepods implications of behaviour. - Marine Ecology Progress Series 131:315-316.

Aksnes, D.L., J. Aure, S. Kaartvedt, T. Magnesen \& J. Richard 1989. Significance of advection for the carrying capacities of fjord populations. - Marine Ecology Progress Series 50:263-274.

Aksnes, D.L. \& J. Blindheim 1996. Circulation patterns in the North Atlantic and possible impact on population dynamics of Calanus finmarchicus. Ophelia 44:7-28.

Aksnes, D. L. \& T. Høisæter 1987. Obtaining life table data from stage-frequency distributional statistics. - Limnology and Oceanography 32:514-517. 
Aksnes, D.L. \& T. Magnesen 1983. Distribution, development, and production of Calanus finmarchicus in LindEspollene, western Norway, 1979. - Sarsia 68:195-208.

- 1988. A population dynamics approach to the estimation of production of four copepods in Lindåspollene, western Norway. - Marine Ecology Progress Series 45:57-68

Aksnes, D.L. \& M.D. Ohman 1996. A vertical life table approach to zooplankton mortality estimation. Limnology and Oceanography 41:1461-1469.

Banks, H.T.L., W. Botsford, F. Kappel \& C. Wang 1991. Estimation of growth and survival in size-structured cohort data - an application to larval stripped bass (Morone saxatilis). - Journal of Mathematical Biology 30:125-150.

Belehrádek, J. 1935. Temperature and living matter. Protoplasma Monograph No. 8. - Borntraeger, Berlin. 277 pp.

Brett, M.T.L., Martin \& T. Kawecki 1992. An experimental test of the egg ratio method: estimated versus observed death rates. - Freshwater Biology 28:237-248.

Burkill, P.H. \& T.F. Kendall 1982. Production of the copepod Eurytemora affinis in the Bristol Channel. - Marine Ecology Progress Series 7:21-31.

Carlotti, F. \& S. Nival 1992. Moulting and mortality rates of copepods related to age within stage: experimental results. - Marine Ecology Progress Series 84:235-243.

Caswell, H. 1989. Matrix population models: construction, analysis, and interpretation. - Sinauer Associates, Inc. Publishers Sunderland, Massachusetts. 328 pp.

Caswell, H. \& S. Twombly 1989. Estimation of stage-specific demographic parameters for zooplankton populations: Methods based on stage-classified matrix projection models. - Lecture Notes in Statistics 55:93-107.

Corkett, C.J. 1967. Technique for rearing marine calanoid copepods in laboratory conditions. - Nature 216:5859.

Corkett, C.J., I.A. McLaren, J.-M. Sevigny 1986. The rearing of calanoid copepods Calanus finmarchicus, $C$. glacialis Jaschnov and C. hyperboreus Kroyer with comment on the equiproportional rule. - National Museums of Canada, Syllogeus Series 58:539-546.

Cushing, D.H. \& D.S. Tungate 1963. Studies of a Calanus patch. I. The identification of a Calanus patch. Journal of Marine Biological Association of United Kingdom 43:327-337.

DeAngelis, D.L. \& L. Gross (eds.) 1992. Individual-based models and approaches in ecology. - Chapman and Hall. 525 pp.

Durbin, A.G. \& E.G. Durbin 1981. Standing stock and estimated production rates of phytoplankton and zooplankton in Narragansett Bay, Rhode Island. Estuaries 4:24-41.

Edmondson, W.T. 1960. Reproductive rates of rotifers in natural populations. - Memorie dell' Istituto Italiano di Idrobiologia 12:21-77.

- 1993. Experiments and quasi-experiments in limnology. - Bulletin of Marine Science 53:65-83
Edmondson, W.T. \& G.G. Winberg 1971. A manual on methods for assessment of secondary production in fresh waters. - Blackwell Scientific Publications, Oxford. $358 \mathrm{pp}$.

Fager, E.W. 1973. Estimation of mortality coefficients from field samples of zooplankton. - Limnology and Oceanography 18:297-301.

Fasham, M.J.R. 1993. Modelling the marine biota. - Pp. 457-504 in: M. Heidmann (ed.). The global carbon cycle. Springer Verlag, New York. 599 pp.

Gabriel, W., B.E. Taylor \& S. Kirsch-Prokosh 1987. Cladoceran birth and death rate estimates: experimental comparisons of egg ratio methods. Freshwater Biology 18:361-372.

Gehrs, C.W. \& A. Robertson 1975. Use of life tables in analyzing the dynamics of copepod populations. Ecology 56:665-673.

Gill, P.E., W. Murray, \& M.H. Wright 1981. Practical optimization. - Academic Press, London. 401 pp.

Gliwicz, Z.M. \& A. Rykowska 1992. "Shore avoidance" in zooplankton: a predator-induced behaviour or predator-induced mortality. - Journal of Plankton Research 14:1331-1342.

Gurney, W.S.C., R.M. Nisbet, \& S.P. Blythe 1986. The systematic formulation of models of stage-structured populations. - Pp. 475-459 in: Metz, J.A.J. \& O. Diekmann (eds). The dynamics of physiologically structured populations. Lecture Notes in Biomathematics 68. Springer-Verlag, Berlin.

Hairston, N.G. \& S. Twombly 1985. Obtaining life table data from cohort analysis: a critique of current methods. Limnology and Oceanography 30:886-893.

Hay, S.J., G.T. Evans, \& J.C. Gamble 1988. Birth, death and growth rates for enclosed populations of calanoid copepods. - Journal of Plankton Research 10:431-454.

Heinle, D.R. 1966. Production of a calanoid copepod, Acartia tonsa, in the Patuxcent River estuary. Chesapeake Science 7:59-74.

Herman, A.W., D.D. Sameoto, C. Shunnian, M.R. Mitchell, B. Petrie, \& N. Cochrane 1991. Sources of zooplankton on the Nova Scotia Shelf and their aggregations within deep-shelf basins. - Continental Shelf Research 11:211-238.

Heron, A.C. 1972. Population ecology of a colonizing species: The pelagic tunicate Thalia democratica. II. Population growth rate. - Oecologia 10:294-312.

Huntley, M.E. \& M.D.G. Lopez 1992. Temperature-dependent production of marine copepods: a global synthesis. - American Naturalist 140(2):201-242.

Ianora, A. \& I. Buttino 1990. Seasonal cycles in population abundances and egg production rates in the planktonic copepods Centropages typicus and Acartia clausi. - Journal of Plankton Research 12:473-481.

Kaartvedt, S. 1993. Drifting and resident plankton. - Bulletin of Marine Science 53:154-159.

Ketchum, B.H. 1954. Relation between circulation and planktonic populations in estuaries. - Ecology 35:191-200.

Kimmerer, W. J. \& A. D. McKinnon 1987. Growth, mortality and secondary production in the copepod Acartia tranteri in Westernport Bay, Australia. - Limnology and Oceanography 32:14-28. 
Kiørboe, T., F. Møhlenberg \& P. Tiselius 1988. Propagation of planktonic copepods: production of eggs. Hydrobiologia 167/168:219-225.

Kiørboe, T. \& T.G. Nielsen 1994. Regulation of zooplankton biomass and production in a temperate, coastal ecosystem. I. Copepods. - Limnology and Oceanography 39:493-507.

Klein Breteler, W.C.M., N. Schogt \& J. van der Meer 1994. The duration of copepod life stages from stage-frequency data. - Journal of Plankton Research 16:1039-1057.

Landry, M.R. 1978. Population dynamics and production of a planktonic marine copepod, Acartia clausi, in a small temperate lagoon on San Juan Island, Washington. - Internationale Revue der gesamten Hydrobiologie 63:77-119.

McLaren, I.A. 1963. Effects of temperature on growth of zooplankton, and the adaptive value of vertical migration. - Journal of the Fisheries Research Board of Canada 20:685-727.

McLaren, I.A., Sévigny, J.-M. \& Corkett, C.J. 1989. Temperature dependent development in Pseudocalanus species. - Canadian Journal of Zoology 67:559-564

Manly, B.J.F. 1974. Estimation of stage-specific survival rates and other parameters for insect populations developing through several stages. - Oecologia 15:277285.

- 1977. A further note on Kiritani and Nakasuji's model for stage frequency data including comments on the use of Tukey's jackknife technique for estimating variances. - Researches on Population Ecology 18:177-186.

- 1985. Further improvements to a method for analyzing stage-frequency data. - Researches on Population Ecology 27:325-332.

- 1990. Stage-structured populations: Sampling, analysis and simulation. - Chapman and Hall Ltd, London. 187 pp.

Matthews, J.B.L., L. Hestad \& J.L.W. Bakke 1978. Ecological studies in Korsfjorden, Western Norway. The generations and stocks of Calanus hyperboreus and C. finmarchicus 1971-1974. - Oceanologia acta $1: 274-284$

Metz, J.A.J, \& O. Diekmann 1986. The dynamics of physiologically structured populations. - Lecture Notes in Biomathematics 68. Springer-Verlag, Berlin. 511 pp.

Miller, C.B. 1993. Development of large copepods during spring in the Gulf of Alaska. - Progress in Oceanography 32:295-317.

Miller, C.B., M.E. Huntley \& E.R. Brooks 1984. Postcollection molting rates of planktonic marine copepods: measurement, applications, problems. Limnology and Oceanography 29:1274-1289.

Miller, C.B. \& D.C. Judkins 1981. Design of pumping systems for sampling zooplankton, with description of two high-capacity samplers for coastal studies. - Biological Oceanography 1:29-56.

Miller, C.B. \& R.D. Nielsen 1988. Development and growth of large, calanoid copepods in the ocean[ic] subarctic Pacific, May 1984. - Progress in Oceanography 20:275-292.
Miller, C.B. \& K.S. Tande 1993. Stage duration estimation for Calanus populations, a modelling study. - Marine Ecology Progress Series 102:15-34.

Mullin, M.M. \& E.R. Brooks 1967. Laboratory culture, growth rate, and feeding behavior of a planktonic marine copepod. - Limnology and Oceanography 12:657-666.

- 1970. Production of the planktonic copepod Calanus helgolandicius. - Bulletin Scripps Institution of Oceanography 17:89-103.

- 1976. Some consequences of distributional heterogeneity of phytoplankton and zooplankton. Limnology and Oceanography 21:784-796.

Ohman, M.D. 1986. Predator-limited population growth of the copepod Pseudocalanus sp. - Journal of Plankton Research 8:673-713.

Ohman, M.D., D.L. Aksnes \& J. Runge 1996. The interrelationship of copepod fecundity and mortality. Limnology and Oceanography 41:1470-1477.

Ohman, M.D. \& S.N. Wood 1995. The inevitability of mortality. - ICES Journal of Marine Science 52:517-522.

1996. Mortality estimation for planktonic copepods: Pseudocalanus newmani in a temperate fjord. - Limnology and Oceanography 41:126-135.

Osgood, K. E. \& B. W. Frost 1996. Effects of advection on the seasonal abundance patterns of three species of planktonic calanoid copepods in Dabob Bay, Washington. - Continental Shelf Research 16:1225-1243.

Paloheimo, J.E. 1974. Calculation of instantaneous birth rate. - Limnology and Oceanography 19:692-694.

Parslow, J., N.C. Sonntag \& J.B.L. Matthews 1979. Technique of systems identification applied to estimating copepod population parameters. - Journal of Plankton Research 1:137-151.

Peterson, W.T. \& W.J. Kimmerer 1994. Processes controlling recruitment of the marine calanoid copepod Temora longicornis in Long Island Sound: Egg production, egg mortality, and cohort survival rates. Limnology and Oceanography 39:1594-1605.

Peterson, W.T. \& S.J. Painting 1990. Developmental rates of the copepods Calanus australis and Calanoides carinatus in the laboratory, with discussion of methods used for calculation of development time. - Journal of Plankton Research 12:283-293.

Peterson, W.T., P. Tiselius \& T. Kiørboe 1991. Copepod egg production, moulting and growth rates, and secondary production, in the Skagerrak in August 1988. - Journal of Plankton Research 13:131-154.

Press, W.H., B.P. Flannery, S.A. Teukolsky \& W.T. Vetterling 1988. Numerical recipes in C. - Cambridge University Press, Cambridge. 735 pp.

Rigler, F.H. \& J.M. Cooley 1974. The use of field data to derive population statistics of multivoltine copepods. - Limnology and Oceanography 19:636-655.

Sinclair, M. 1988. Marine populations: an essay on population regulation and speciation. - Wahington Sea Grant, Seattle, 252 pp.

Sonntag, N.C. \& J. Parslow 1981. Technique of systems identification applied to estimating copepod production, Journal of Plankton Research 3:461-473. 
Taylor, B.E. \& M. Slatkin 1981. Estimating birth and death rates of zooplankton. - Limnology and Oceanography 26:143-158.

Threlkeld, S.T. 1979. Estimating cladoceran birth rates: The importance of egg mortality and the egg age distribution. - Limnology and Oceanography 24:601-612.

Twombly, S. 1994. Comparative demography and population dynamics of two coexisting copepods in a Venezuelan flood plain lake. - Limnology and Oceanography 39:234-247.

Tyler, J.A. \& K.A. Rose 1994. Individual variability and spatial heterogeneity in fish population models. Reviews in Fish Biology and Fisheries. 4:91-123.

Uye, S.-I. 1982. Population dynamics and production of Acartia clausi Giesbrecht (Copepoda: Calonoida) in inlet waters. - Journal of Experimental Marine Biology and Ecology 57:55-83.
Wood, S.N. 1994. Obtaining birth and mortality patterns from structured population trajectories. - Ecological Monographs 64:23-44.

Wood, S.N., S.P. Blythe, W.S.C. Gurney, \& R.M. Nisbet 1989. Instability in mortality estimation schemes related to stage structured population models. - IMA Journal of Mathematics Applied in Medicine and Biology. 6:47-68.

Wood, S.N. \& R.M. Nisbet 1991. Estimation of mortality rates in stage structured populations. Lecture Notes in Biomathematics 90. Springer-Verlag, Berlin. 101 pp.

Zillioux, E.J. \& D.F. Wilson 1966. Culture of a planktonic calanoid copepod through multiple generations. Science 151:996-998.

Accepted 23 June 1997 